Research Article

\title{
Finite Element Model Updating in Bridge Structures Using Kriging Model and Latin Hypercube Sampling Method
}

\author{
Jie Wu $\mathbb{D}^{1},{ }^{1}$ Quansheng Yan, ${ }^{1}$ Shiping Huang $\left(\mathbb{D},{ }^{1,2}\right.$ Chao Zou, ${ }^{3}$ Jintu Zhong $\mathbb{D}^{1}$ \\ and Weifeng Wang (iD 1 \\ ${ }^{1}$ School of Civil Engineering and Transportation, South China University of Technology, Guangzhou 510640, China \\ ${ }^{2}$ State Key Laboratory of Coal Resources and Safe Mining, China University of Mining and Technology, Xuzhou 221116, China \\ ${ }^{3}$ School of Civil and Transportation Engineering, Guangdong University of Technology, Guangzhou 510006, China
}

Correspondence should be addressed to Shiping Huang; ctasihuang@scut.edu.cn and Weifeng Wang; ctwfwang@scut.edu.cn

Received 7 September 2018; Revised 19 November 2018; Accepted 24 November 2018; Published 11 December 2018

Academic Editor: Filippo Ubertini

Copyright ( 2018 Jie Wu et al. This is an open access article distributed under the Creative Commons Attribution License, which permits unrestricted use, distribution, and reproduction in any medium, provided the original work is properly cited.

Computational cost reduction and best model updating method seeking are the key issues during model updating for different kinds of bridges. This paper presents a combined method, Kriging model and Latin hypercube sampling method, for finite element (FE) model updating. For FE model updating, the Kriging model is serving as a surrogate model, and it is a linear unbiased minimum variance estimation to the known data in a region which have similar features. To predict the relationship between the structural parameters and responses, samples are preselected, and then Latin hypercube sampling (LHS) method is applied. To verify the proposed algorithm, a truss bridge and an arch bridge are analyzed. Compared to the predicted results obtained by using a genetic algorithm, the proposed method can reduce the computational time without losing the accuracy.

\section{Introduction}

Bridge structures are playing important roles in our lives for supporting essential social and economic functionalities. However, they are potentially prone to damage due to significant loads during their service lives. For this reason, structural health monitoring (SHM) technologies have been deeply studied with the goal to monitor the conditions of the bridges and detect the damages as well as assess their conditions [1]. As a result, high accuracy FE models of bridges are often critical to structural condition evaluations. However, the initial FE model is established based on the design drawings which does not take environmental effects and other unexpected conditions into consideration, thus some inevitable differences occur, and modelling errors always exist in FE models. While there are many methods proposed to accommodate the errors, it is critical to reduce modelling errors through FE model updating by calibrating structural model parameters based on measured data [2].

For a long time, algorithm development for FE model updating is a hot research area in SHM, and the goal of FE model updating is to minimize the discrepancies between measured data of a real structure and FE model [3]. Until now, FE model updating methods can be divided into two groups. One is called the direct method, which updates the elements of mass and stiffness matrix. The other is the iteration method, which updates the structural parameters iteratively to minimize the differences between the measured data and the analytical response values. For the direct method, the updated mass or stiffness matrixes usually lose the sparse character, and some elements do not maintain corresponding physical meaning [4]. In order to overcome the drawbacks, many researchers proposed different methods. Kabe [5] used the structural connective information to optimally adjust deficient stiffness matrices and obtained the excellent results. By utilizing the added known masses, new mass and stiffness updating algorithms were proposed by $\mathrm{Cha}$ and $\mathrm{Gu}$ [6]. Their method preserved the physical configuration of the analytical model and reduced the computational efforts. Minas and Inman [7] used an eigenstructure assignment algorithm to calculate corrections by the FE model, and the results from an updated FE model 
agreed with experimental data. Based on the development of a new symmetric eigenstructure assignment method, Zimmerman and Widengren [8] used the incorporated measured experimental modal data to update an FE model. However, the mentioned methods have the limitation that the updated structural matrices cannot feedback to the general FE model software. Compared to the direct method, parametric methods are widely used by selecting specific parameters to update the FE model to obtain the minimum error between the updated model and experimental data.

For complex structures, such as cable-stayed bridges, suspension bridges, and the other composite bridges, the number of nodes, elements, materials, and boundary conditions are complex.

It is still an issue to obtain accurate results with less computational time. To achieve high computational efficiency, some studies on substructure methods are accounted for truncation approximation method proposed by Weng et al. [9]. The improved computation efficiency of the method was verified by a frame structure and a practical bridge with the lowest eigensolutions of substructures. However, this kind of method lacks principles for substructure division, which affects the results of the updated FE model. Then, the focus of researchers turned to the surrogate model method, which considers the regression analysis and experiment design. It established the explicit function to model the relationship between the structural responses and the parameters of structures and then applied the explicit function to the FE model to get the analytical data [10]. Marwala [11] proposed the response surface method for FE model updating, using genetic algorithm (GA) to optimize the selected parameters and verified the computational efficiency of the proposed method by an unsymmetrical $\mathrm{H}$-shaped structure. By using response surface method, Ren and Chen [12] accounted for sampling with a design of experiments, selecting the significant updating parameters and constructing polynomial response surface, and then a novel method was proposed and its application to a full-size bridge had been demonstrated efficiently. Furthermore, Shan et al. [13] proposed an FE model updating method, which combined the substructure and response surface model updating method together. The proposed method was applied to a cable-suspension bridge, and the efficiency of the method was verified. As response surface method and its related methods are almost constructed on training samples which include input and output information of the system, researchers have been motivated to tackle the challenge from the perspective of the Kriging model. The theory of the Kriging model is based on a correlation function, which is an interpolation of the known data and can go through all the sampling points. Compared to traditional response surface method, the Kriging model has much higher approximation accuracy.

For exploring Kriging model updating in bridge structures, lots of researchers play important roles during the development of this method. In order to select the inputs to predict efficiently, Sacks et al. introduced the Kriging model, which originated from a statistical approach to mine valuation by a South African geologist Krige [14, 15]. Based on progressive lattice sampling, Romero et al. constructed several efficiently upgradable response surface approximations. Compared to other interpolation schemes, the Kriging model almost performed best [16]. Zhang and Au [17] applied the Kriging model in initial cable forces calibration, and the advantages of feasibility and accuracy of the Kriging model were verified. To balance the accuracy and efficiency of implementing reliability analysis, Zhang et al. [18] proposed an advanced Kriging method to combine with simple random sampling method and leave-one-out technique as well as Monte Carlo simulation method, and the accuracy and efficiency of the proposed method were verified through some engineering examples. Huang et al. [19] proposed an active learning method which combined the Kriging model and subset simulation to do small failure probability assessment. By applying the proposed method to several benchmark functions, the advantage of providing accurate solutions much more efficiently was demonstrated. Based on frequency response function, Wang et al. [20] updated the model with the Kriging model.

This paper uses the Kriging model updating for a truss bridge and an arch bridge. The training points for the Kriging model are obtained by Latin hypercube Sampling (LHS) instead of the design of an experiment. For these the two cases, the acceleration data were measured and used for optimization. LHS is applied to produce training samples. Compared to genetic algorithm (GA), the model updating results of two cases showed that the proposed method has significant improvement to reduce the calculation time without losing the accuracy.

\section{Methodology}

2.1. Kriging Model. The Kriging model was proposed by a geologist D. G. Krige. When applied it as a surrogate model, the response function $y(x)$ consists of two parts. One is a regression model $F(\beta, x)$, and the other is stochastic process $z(x)[21-23]:$

$$
\begin{aligned}
y(x) & =F(\beta, x)+z(x), \\
F(\beta, x) & =\beta_{1} f_{1}(x)+\beta_{2} f_{2}(x)+\cdots+\beta_{p} f_{p}(x) \\
& =\left[f_{1}(x), \ldots, f_{p}(x)\right] \beta \\
& =f(x)^{T} \beta,
\end{aligned}
$$

where $f(x)^{T}=\left[f_{1}(x), \ldots, f_{p}(x)\right]^{T}$ is the trend function which consists of a vector of regression function. $\beta=\left[\beta_{1}, \ldots, \beta_{p}\right]^{T}$ is the corresponding vector of unknown regression coefficients, and $p$ is the number of $f_{i}(x)$. The term $z(x)$ is the realization of a stochastic process with zero mean, $\sigma^{2}$ variance, and nonzero covariance. The covariance matrix of $z(x)$ can be described as

$$
\operatorname{cov}\left[z\left(x_{i}\right), z\left(x_{j}\right)\right]=\sigma^{2} R\left(\theta, x_{i}, x_{j}\right), \quad i, j=1,2, \ldots, n,
$$

where $n$ denotes the number of the experiment points. $R\left(\theta, x_{i}, x_{j}\right)$ is the correlation function between $x_{i}$ and $x_{j}$ and defined by its set of parameters $\theta[22,24]$. The correlation 
function needs to be selected by users. In this paper, $R\left(\theta, x_{i}, x_{j}\right)$ is defined by Gaussian spatial correlation function $[25,26]$ :

$$
R\left(x_{i}, x_{j}\right)=\exp \left(-\sum_{k=1}^{p} \theta_{k}\left|x_{i}^{k}-x_{j}^{k}\right|^{2}\right),
$$

where $\theta_{k}$ is unknown correlation parameter utilized to fit the model [27] and $x_{i}^{k}$ and $x_{j}^{k}$ are the $k$ th components of sample points $x_{i}$ and $x_{j}$.

In the Kriging model, the values of $\mu, \sigma^{2}$, and $\theta$ are determined by maximizing the likelihood function. First, $\theta$ is obtained by minimizing the concentrated log-likelihood function as [28]

$$
\max _{\theta_{k}>0}\left[-\frac{n \ln \left(\sigma^{2}\right)+\ln |R|}{2}\right], \quad k=1,2, \ldots, n .
$$

$\beta$ and $\sigma^{2}$ can be estimated as [29]

$$
\begin{aligned}
\widehat{\beta} & =\left(f^{T} R^{-1} f\right)^{-1} f^{T} R^{-1} y, \\
\widehat{\sigma}^{2} & =\frac{1}{n}(y-f \widehat{\beta})^{T} R^{-1}(y-f \widehat{\beta}) .
\end{aligned}
$$

Then, the prediction value $\hat{y}(x)$ for the response $y(x)$ at an untried value of $x$ can be obtained as

$$
\hat{y}(x)=f^{T}(x) \hat{\beta}+r^{T}(x) R^{-1}(Y-f \widehat{\beta}),
$$

where $\hat{\beta}$ is the estimated value of $\beta$, $r^{T}(x)=\left[r\left(x, x_{1}\right), \ldots, r\left(x, x_{n}\right)\right]$ is the correlation vector evaluated at $x$, and $Y$ is the vector or responses to the sample locations $\left\{x_{1}, x_{2}, \ldots, x_{n}\right\}$ [30].

Above equation is equivalent to the minimum optimization problem:

$$
\min _{\theta_{k}>0}\left(|R|^{1 / p} \sigma^{2}\right) .
$$

Finally, $\theta_{k}$ can be obtained, and the Kriging model can be also constructed.

2.2. Latin Hypercube Sampling Method. For FE model updating, one key issue is to obtain several sample points. The sampling methods mainly can be classified as simple random sampling (SRS), stratified sampling method, cluster sampling method, and systematic sampling. For SRS, it is an unbiased surveying technique and a subset of individuals chosen from a large set [31]. For stratified sampling, it is a method of sampling from a population [32]. Cluster sampling is a sampling plan used when mutually homogeneous, yet internally heterogeneous groupings are evident in a statistical population and often used in marketing research [33]. Systematic sampling is a statistical method involving the selection of elements from an ordered sampling frame [34]. SRS is a basic type of sampling since it can be a component of other more complicated sampling methods. However, when a complete sampling frame is not available or not much information about the population is available, SRS is not a good choice. In this case, stratified sampling may be a better choice.
Latin hypercube sampling (LHS) is a stratified sampling technique proposed by Mckay et al. [35]. It was developed by W. J. Conover in the summer of 1975 when he was a consultant. It was further elaborated by Iman et al. in 1981 [36]. It has been widely used in many fields, such as risk assessment, hurricane loss modelling, nuclear power safety assessment, and manufacturing equipment reliability analyses [37].

In LHS, it must be decided how many sample points are to be used, and for each sample point, it is important to remember in which row and column the sample point was taken. LHS can ensure that the ensemble of random numbers is representative of the real variability whereas random sampling is just an ensemble of random numbers without any guarantees.

For LHS, it can take all the variation of input variables into consideration, just with a small number of runs. The total range of the input variables is ensured to be represented. For example, if we want to sample $m$ points in the $n$ dimensional vector space $D \in R^{n}$, it should be done as follows [38]:

(1) Divide the interval of each dimension into $m$ nonoverlapping intervals, and each interval has equal probability (take a uniform distribution, and the intervals should have the equal size)

(2) Sample randomly from a uniform distribution, a point in each interval in each dimension

(3) Pair randomly (equal likely combinations) the points from each dimension

Figure 1 is prepared to show the advantage of LHS. In Figure 1, 10 samples of two random variables obtained by two methods are shown, one is simple random sampling (SRS), and the other is LHS method. It is easy to find that the results of LHS method are much more spread out and have no clustering effects compared to the result of SRS method.

\section{Experimental Verifications}

3.1. Experimental Verification of a Truss Bridge. In this example, we apply the Kriging model to a plane truss to do model updating [39]. The elevation view of the truss bridge is shown in Figure 2. All the members are steel tubes. The inner and outer diameter of each member is $5.4 \mathrm{~cm}$ and $8.5 \mathrm{~cm}$, respectively. Initial elastic modulus is $E=2.1 \times 10^{11} \mathrm{~Pa}$, initial mass density is $\rho=8.1 \times 10^{3} \mathrm{~kg} / \mathrm{m}^{3}$, and Poisson's ratio is 0.3 .

In the literature [39], FE model updating is based on genetic algorithm (GA), and the Kriging model is applied to the structure. The procedure based on GA is given as follows, and a flowchart of the method is shown in Figure 3:

(1) Establish the FE model through ANSYS [40] FE model analysis program. There are 28 nodes, each node has two degrees of freedom (DOF), with 53 DOFs in total.

(2) Assume the damping ratio is $1 \%$ and excite the structure at node 3 with vertical excitation, and the 


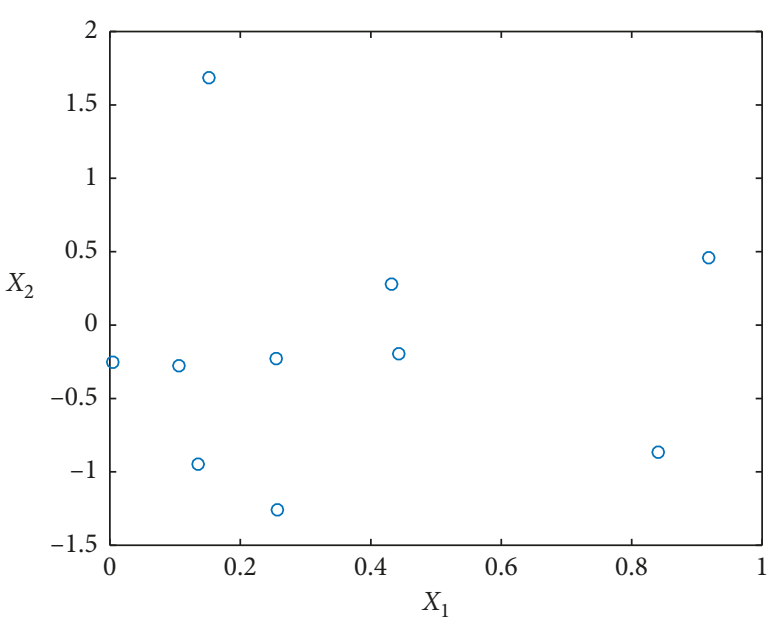

(a)

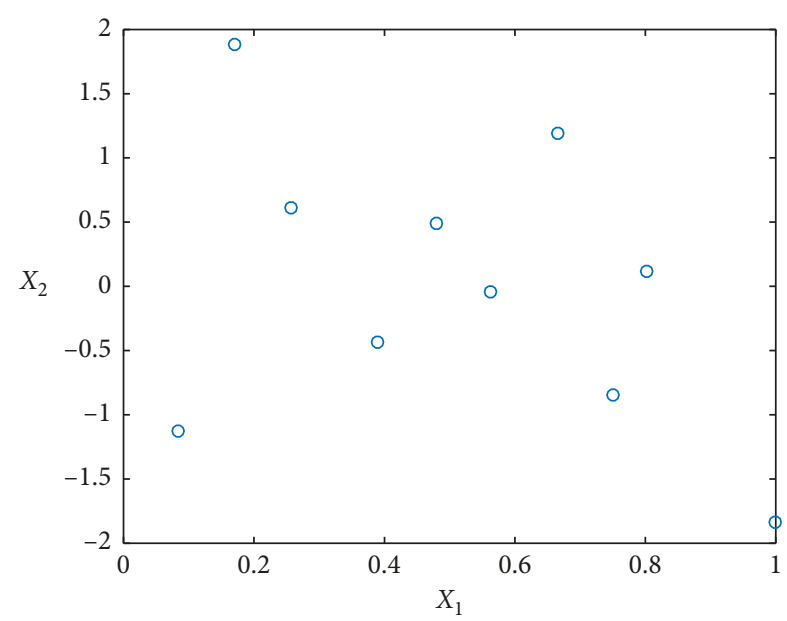

(b)

Figure 1: Examples of two methods to generate a sample of size $n=10$ from two variables $X=\left[X_{1}, X_{2}\right]$ where $X_{1}$ has a uniform distribution $u[0,1]$ and $X_{2}$ has a normal distribution $N[0,1]$. (a) Simple random sampling. (b) Latin hypercube sampling.

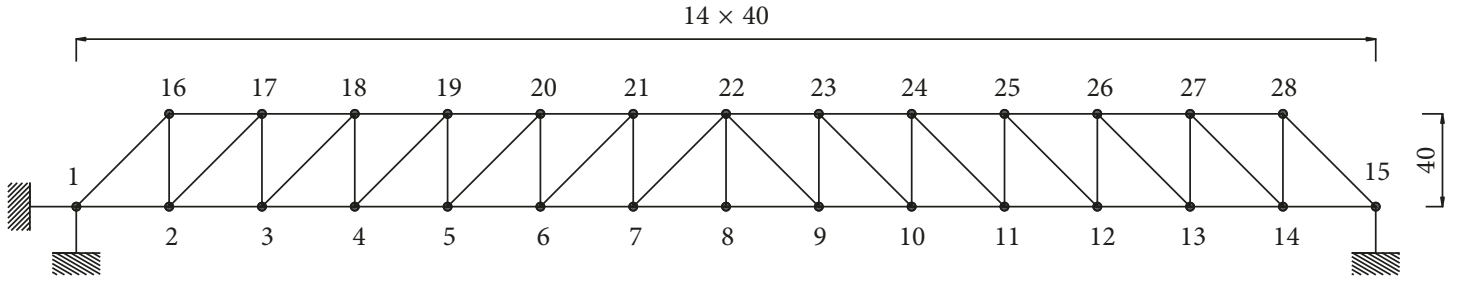

FIGURE 2: Elevation view of the truss bridge (unit: $\mathrm{cm}$ ).

excitation force is simulated with band-limited white noise (BLWN). The vertical acceleration response of each DOF of the structure was calculated by Newmark-beta time-history analysis method. Eigensystem realization algorithm (ERA) was applied to obtain the first sixth modal parameters.

(3) The objective function is established and then optimized by GA. The number of populations is 100 . Then, the updated results are shown in Table 1.

When applying the Kriging model to do model updating, the procedure is as follows, and the flowchart of the Kriging model is shown in Figure 4:

(1) Set elastic modulus and density of the materials as design parameters. Assume these design parameters and follow normal distributions $X \sim N(\mu, \sigma)$, where $\mu$ is the design value for the design parameters, which is the initial value; the standard deviation of variables is $\sigma(\sigma=\mu \times \alpha)$, and $\alpha$ is the variation coefficient of design parameters.

(2) According to the distribution range of each random variable, the initial training samples are obtained through LHS method, and the training samples can be ranged from 10 to 100 .

(3) With the training samples, the structural responses are obtained with the FE model, and then the mapping relation of input-output of the initial training samples is obtained.
(4) The parameter $\theta$ in the Kriging model is optimized based on GA, and the Kriging model is established after the optimization.

(5) Based on the Kriging model, the prediction is performed on the test samples to obtain the corresponding modal parameters.

The comparison optimization results between the GA method in the literature [39] and the Kriging model in this paper are shown in Table 1.

The frequencies of the first 3 modes from the Kriging model are better than that of the GA from the literature, and the errors for the first 6 modes are all below $2 \%$. Based on the updated FE model, the updated design parameters are $E=$ $1.92 \times 10^{11} \mathrm{~Pa}$ and $\rho=8.0241 \times 10^{3} \mathrm{~kg} / \mathrm{m}^{3}$.

3.2. Experimental Verification of a Tied-Arch Bridge. In this example, the FE model of a tied-arch bridge is taken as an object. This bridge is a long-span concrete-filled steel tubular continuous girder arch composite system bridge with the span length of $58.4+128.0+58.4 \mathrm{~m}$ (Figure 5 ). The beam is prestressed concrete structure. The arch rib is a concretefilled steel tubular structure, which is designed as a dumbbell-shaped section with equal height, and the section height is $2.8 \mathrm{~m}$. The arch rib chord and the plate of the bridge are filled with microexpansion concrete. The design vector height is $25.6 \mathrm{~m}$, and the calculated span of the arch rib is $128 \mathrm{~m}$. In total, there are 14 pairs of suspenders, and each of 


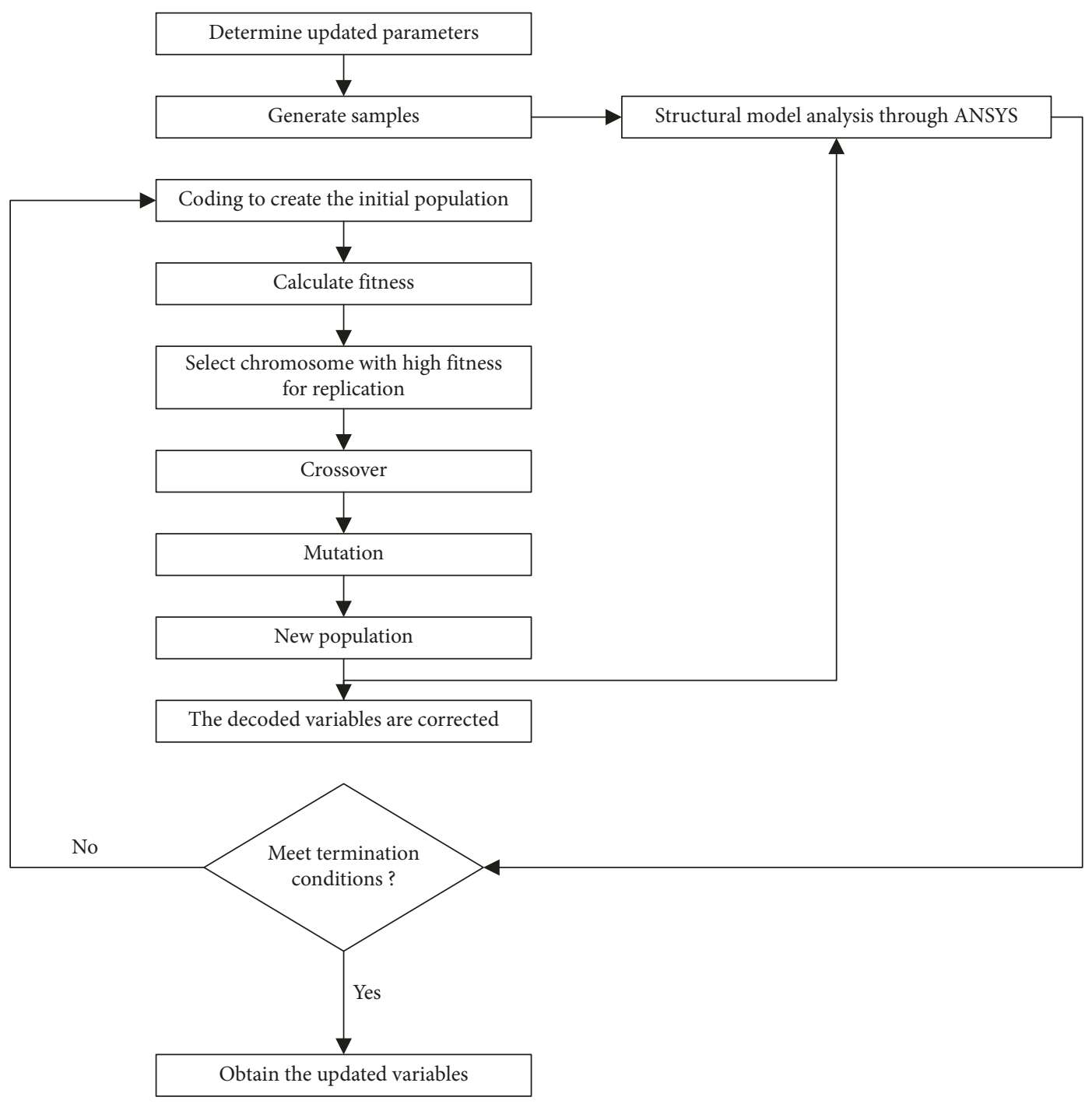

FIgURE 3: Flowchart of GA.

TABLe 1: Comparison of truss updating results (unit: $\mathrm{Hz}$ ).

\begin{tabular}{|c|c|c|c|c|c|}
\hline Mode number & Test & Literature [39] & Error (\%) & Kriging model & Error (\%) \\
\hline 1 & 8.79 & 8.83 & -0.55 & 8.79 & 0.00 \\
\hline 2 & 29.60 & 30.18 & -1.48 & 29.78 & -0.60 \\
\hline 3 & 43.39 & 41.65 & 2.23 & 42.66 & 1.69 \\
\hline 4 & 59.10 & 59.62 & -0.24 & 59.55 & -0.75 \\
\hline 5 & 90.62 & 91.34 & -0.40 & 91.09 & -0.52 \\
\hline 6 & 119.81 & 120.84 & -0.11 & 120.86 & -0.88 \\
\hline
\end{tabular}

them is in the form of double sling with the uniform diameter $(85 \mathrm{~mm})$.

The ambient arbitrary vibration measurement was conducted on the bridge after the bridge construction and before its opening to the operation to obtain the eigenmodes (including natural frequencies, mode shapes, and damping ratios), which were very important parameters for health monitoring and malfunctioning diagnosis of bridges.

In order to obtain the vertical and lateral modal characteristics of the bridge, measuring points are arranged. For the vertical test of the girder, there are 28 sensors, and each of them is arranged on each boom of the girder. For the lateral test of the girder, 7 sensors are arranged, and each of them is placed at the boom of the downstream. For the test of arch rib, in total, 32 sensors are arranged, half for vertical test and the half for the lateral test. The sensors are placed at the position of lateral braces. The arrangement of measuring points is shown in Figure 6.

The space bar model of the bridge was established by ANSYS (Figure 7). The girder is simulated by the element beam 44, which can simulate the varying cross sections. Element beam 189 is used to simulate the arch rib, and 


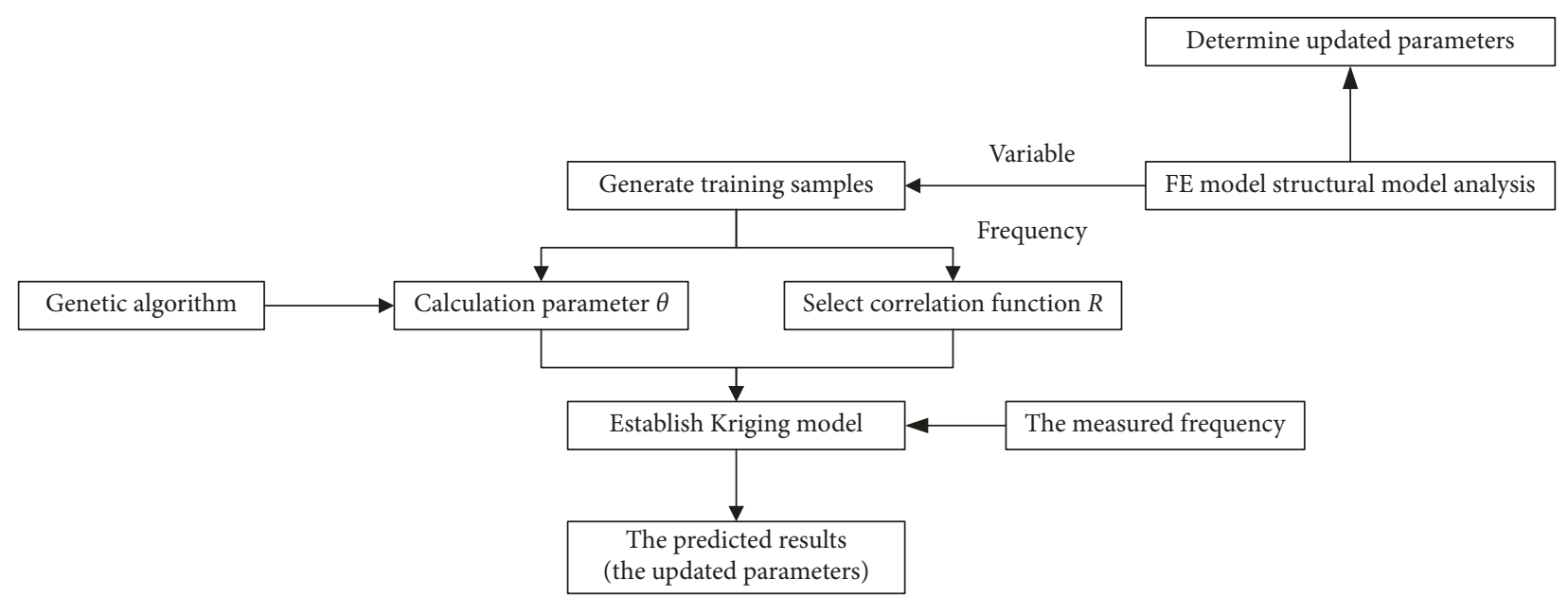

FIgUre 4: Flowchart of the Kriging model.

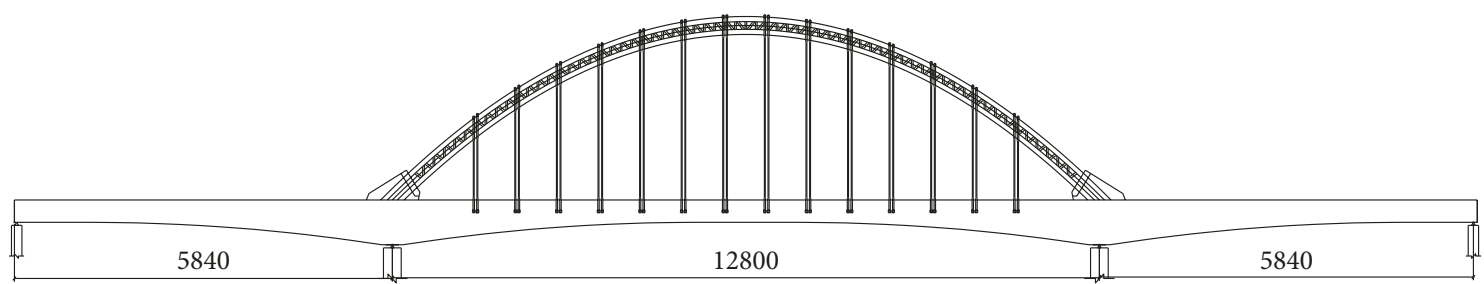

FIgURE 5: Elevation of the bridge (unit: $\mathrm{cm}$ ).

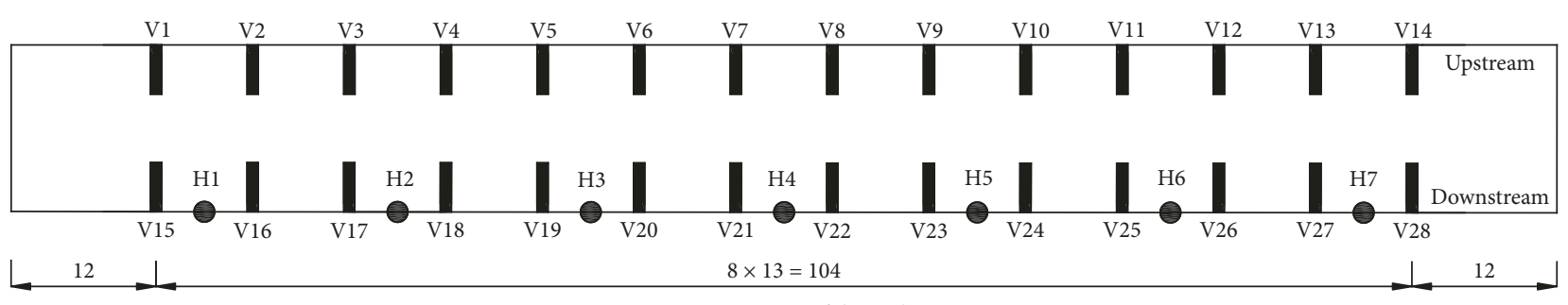

Measuring points of the girder

Vertical measuring point

Lateral measuring point

(a)

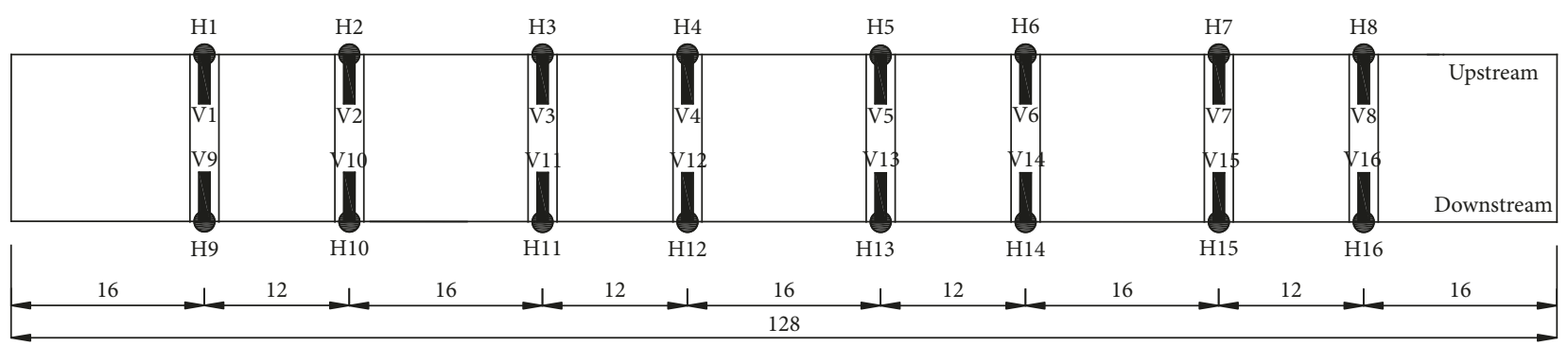

Measuring points of the arch rib

Vertical measuring point

Lateral measuring point

(b)

FIgURE 6: The arrangement of the sensors (unit: $\mathrm{m}$ ). 


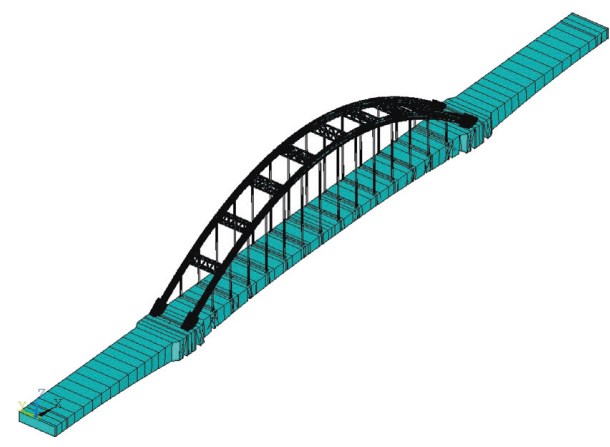

FIgURE 7: The FE model of the bridge.

element link 10 is used to simulate the hanger rod. For the arch rib brace and main arch, girder, and hanger rods, they are connected by rigid arm element.

By using the multiple reference DOF stabilization diagram algorithms based on NExT/ERA (M-NExT/ERA), the modal parameters (natural frequencies and mode shapes) of the bridge are identified. Through the comparison, the results are shown in Table 2.

According to the structural design parameters given in the design drawings, the FE model was established to obtain the original natural frequencies and mode shapes of the bridge. By comparing the results between initial FE model and the experimental results (Table 2), it is obvious to find that for the initial model, in the 17 th model, almost $65 \%$ of their natural frequency errors exceeded $10 \%$, indicating that there is a big difference between the initial FE model and the real structure.

As the initial FE model cannot show the responses of the real structure, in order to ensure the accuracy of the prediction and analysis of the FE model, model updating is necessary. For this arch bridge, the Kriging model and GA are applied.

In the FE model updating, there are many candidate parameters that could be used to produce the required change. One way is to allow all the parameters to take part in the updating procedure, which could lead to expensive computational expense. The other is to select a certain number of updating parameters based on the sensitivity. Then, how to select updating parameters and how many should be selected become important issues. Referred to the literature [10], in this engineering project, the FE model updated parameters are as follows:

(1) Arch rib-concrete: elastic modulus $\left(E_{\mathrm{c} 1}\right)$, density $\left(\rho_{\mathrm{cl}}\right)$;

(2) Arch rib-steel tube: elastic modulus $\left(E_{\mathrm{s} 1}\right)$, density $\left(\rho_{\mathrm{s} 1}\right)$;

(3) Hanger rod: elastic modulus $\left(E_{\mathrm{s} 2}\right)$, density $\left(\rho_{\mathrm{s} 2}\right)$;

(4) Girder-concrete: elastic modulus $\left(E_{\mathrm{c} 2}\right)$, density $\left(\rho_{\mathrm{c} 2}\right)$;

(5) Wind support rigid arm: elastic modulus $\left(E_{\mathrm{g} 1}\right)$;

(6) Hanger rod rigid arm: elastic modulus $\left(E_{\mathrm{g} 2}\right)$;

(7) Secondary dead load $(F)$.

Based on the selected parameters, the two updating methods can be applied.
As mentioned above, the Kriging model is a learning theory which is based on small sample statistical learning and forecasting and is regarded as the best optimizing linear unbiased estimate method. Without affecting the accuracy of the results, the time of calculation and analysis is reduced, and the computational efficiency is improved. To apply the Kriging model, firstly, take 100 sets of parameters as the design variables, and then substitute them to the FE model to obtain 100 sets of results. Secondly, take the first two or three corresponding natural frequencies of the arch rib lateral mode, arch rib vertical mode, girder lateral mode, and arch rib torsional mode as training samples. Then, based on the obtained four groups of modified design parameters, the corresponding weight values are assigned according to the accuracy of the modified results, and the final update results are obtained. The weight coefficient is a random number with the sum of 1 and is distributed according to the accuracy.

When applying genetic algorithm (GA), assume the design parameters obey uniform distributions $X \sim U(M(1-\alpha), M(1+\alpha))$, where $M$ is the value of the design parameter and $\alpha$ is the variation coefficient of the design parameters (same as the Kriging model). In order to obtain the updated parameters, firstly, establish the interface between ANSYS and MATLAB. Then, generate the design parameters within a range of specified variables, and the natural frequencies and mode shapes are obtained by substituting the generated design parameters into the FE model. The results show that the order of mode shapes changes with the change of design parameters.

By using the Kriging model and GA, the updated parameters are listed in Table 3.

Based on the updated parameters, the updated natural frequencies and mode shapes are also obtained. The specific results are shown in Table 4.

In order to show the high correlation of mode shapes between the measurement and FE model, for the simplicity of calculation, only some modes are listed in Table 5. In Table 5, the modal assurance criterion (MAC) of arch rib 1st order symmetrical lateral bending, girder 1st order antisymmetrical lateral bending, girder and arch rib 1st order antisymmetrical vertical bending, and arch rib 1st order torsional mode shapes are given. Except for the arch rib 1st order antisymmetrical vertical bending, the MAC of the others is over 0.95 , which shows a high correlation between the model and measured data.

In order to obtain the corresponding frequency of the bridge, the MAC was applied to match the mode shapes (Table 5). Furthermore, error 2-norm of first-order natural frequency is also shown in Table 6.

In Table 6, the frequency error of the updated model by using the Kriging model is the smallest, while the frequency accuracy of the noncorresponding updated model is slightly insufficient. All the errors of updated models with the Kriging model are below $10 \%$, while the error 2-norm of the Kriging model is higher than GA. For this bridge, the calculation time of Kriging model is about 0.2 times that of GA. The FE model updated method based on the Kriging model reduces the dependency on the number of samples and 
TABLe 2: Comparison of testing results and FE model results (unit: $\mathrm{Hz}$ ).

\begin{tabular}{|c|c|c|c|c|}
\hline Measured frequency & FEM frequency & Error (\%) & Position & Modal description \\
\hline 0.5911 & 0.4894 & 17.21 & \multirow{7}{*}{ Arch rib } & 1st order symmetrical lateral bending \\
\hline 1.1804 & 1.0322 & 12.56 & & 2nd order antisymmetrical lateral bending \\
\hline 1.8387 & 1.6145 & 12.19 & & 3rd order symmetrical lateral bending \\
\hline 2.5883 & 2.3091 & 10.79 & & 4th order antisymmetrical lateral bending \\
\hline 3.4136 & 2.9343 & 14.04 & & 5th order symmetrical lateral bending \\
\hline 4.3012 & 3.8008 & 11.63 & & 6th order antisymmetrical lateral bending \\
\hline 5.1021 & 4.5375 & 11.07 & & 7th order symmetrical lateral bending \\
\hline 2.0143 & 2.0799 & -3.26 & \multirow{6}{*}{ Girder and arch rib } & 1st order antisymmetrical vertical bending \\
\hline 2.9367 & 3.0691 & -4.51 & & 2nd order symmetrical vertical bending \\
\hline 3.2499 & 3.5905 & -10.48 & & 3rd order symmetrical vertical bending \\
\hline 3.8289 & 4.2221 & -10.27 & & 4th order antisymmetrical vertical bending \\
\hline 4.7944 & 4.4713 & 6.74 & & 5th order symmetrical vertical bending \\
\hline 4.9524 & 5.0990 & -2.96 & & 6th order antisymmetrical vertical bending \\
\hline 3.9760 & 3.2979 & 17.05 & \multirow{2}{*}{ Girder } & 1st order antisymmetrical lateral bending \\
\hline 4.6262 & 5.2794 & -14.12 & & 2nd order symmetrical lateral bending \\
\hline 4.4507 & 4.6486 & -4.45 & \multirow{2}{*}{ Arch rib } & 1st order torsional \\
\hline 5.4851 & 5.5923 & -1.95 & & 2nd order torsional \\
\hline
\end{tabular}

TABLE 3: Updated results of design parameters.

\begin{tabular}{|c|c|c|c|c|}
\hline Design parameter & Initial model & Kriging model & GA & Variation coefficient (\%) \\
\hline$E_{\mathrm{c} 1}(\mathrm{~Pa})$ & $3.45 E+10$ & $3.51 E+10$ & $3.76 E+10$ & 10 \\
\hline$\rho_{\mathrm{cl}}\left(\mathrm{kgm}^{-3}\right)$ & 2600.00 & 2394.30 & 2537.30 & 10 \\
\hline$E_{\mathrm{s} 1}(\mathrm{~Pa})$ & $2.06 E+11$ & $2.47 E+1$ & $2.58 E+11$ & 5 \\
\hline$\rho_{\mathrm{s} 1}\left(\mathrm{kgm}^{-3}\right)$ & 7800.00 & 7992.40 & 7794.80 & 5 \\
\hline$E_{\mathrm{s} 2}(\mathrm{~Pa})$ & $1.96 E+11$ & $2.10 E+11$ & $1.99 E+11$ & 5 \\
\hline$\rho_{\mathrm{s} 2}\left(\mathrm{kgm}^{-3}\right)$ & 7800.00 & 7738.20 & 7661.60 & 5 \\
\hline$E_{\mathrm{g} 1}(\mathrm{~Pa})$ & $1.50 E+14$ & $1.81 E+14$ & $1.71 E+14$ & 10 \\
\hline$E_{\mathrm{c} 2}(\mathrm{~Pa})$ & $3.45 E+10$ & $3.41 E+10$ & $3.25 E+10$ & 10 \\
\hline$\rho_{\mathrm{c} 2}\left(\mathrm{kgm}^{-3}\right)$ & 2600.00 & 2850.90 & 2603.00 & 10 \\
\hline$E_{\mathrm{g} 2}(\mathrm{~Pa})$ & $1.50 E+14$ & $1.98 E+14$ & $1.83 E+14$ & 10 \\
\hline$F(\mathrm{~N})$ & $1.45 E+06$ & $1.35 E+06$ & $1.45 E+06$ & 8 \\
\hline
\end{tabular}

TABle 4: Comparison of testing results and FE model results (unit: $\mathrm{Hz}$ ).

\begin{tabular}{|c|c|c|c|c|c|c|}
\hline $\begin{array}{l}\text { Measured } \\
\text { frequency }\end{array}$ & $\begin{array}{c}\text { FEM } \\
\text { frequency }\end{array}$ & Error $(\%)$ & $\begin{array}{c}\text { Kriging } \\
\text { model }\end{array}$ & GA & Position & Modal description \\
\hline 0.5911 & 0.4894 & 17.21 & 0.5369 & 0.5408 & \multirow{7}{*}{ Arch rib } & 1st order symmetrical lateral bending \\
\hline 1.1804 & 1.0322 & 12.56 & 1.1307 & 1.1380 & & 2nd order antisymmetrical lateral bending \\
\hline 1.8387 & 1.6145 & 12.19 & 1.7664 & 1.7805 & & 3rd order symmetrical lateral bending \\
\hline 2.5883 & 2.3091 & 10.79 & 2.5228 & 2.5445 & & 4th order antisymmetrical lateral bending \\
\hline 3.4136 & 2.9343 & 14.04 & 3.1978 & 3.2306 & & 5 th order symmetrical lateral bending \\
\hline 4.3012 & 3.8008 & 11.63 & 4.1338 & 4.1753 & & 6 th order antisymmetrical lateral bending \\
\hline 5.1021 & 4.5375 & 11.07 & 4.9215 & 4.9764 & & 7th order symmetrical lateral bending \\
\hline 2.0143 & 2.0799 & -3.26 & 2.0350 & 2.0455 & \multirow{6}{*}{ Girder and arch rib } & 1st order antisymmetrical vertical bending \\
\hline 2.9367 & 3.0691 & -4.51 & 2.9730 & 3.0057 & & 2nd order symmetrical vertical bending \\
\hline 3.2499 & 3.5905 & -10.48 & 3.4377 & 3.4942 & & 3rd order symmetrical vertical bending \\
\hline 3.8289 & 4.2221 & -10.27 & 4.1427 & 4.1815 & & $\begin{array}{c}\text { 4th order antisymmetrical vertical } \\
\text { bending }\end{array}$ \\
\hline 4.7944 & 4.4713 & 6.74 & 4.4020 & 4.4282 & & 5 th order symmetrical vertical bending \\
\hline 4.9524 & 5.0990 & -2.96 & 5.2573 & 5.1614 & & $\begin{array}{c}\text { 6th order antisymmetrical vertical } \\
\text { bending }\end{array}$ \\
\hline 3.9760 & 3.2979 & 17.05 & 3.7637 & 3.8466 & \multirow{2}{*}{ Girder } & 1st order antisymmetrical lateral bending \\
\hline 4.6262 & 5.2794 & -14.12 & 5.0266 & 5.1318 & & 2nd order symmetrical lateral bending \\
\hline 4.4507 & 4.6486 & -4.45 & 4.8588 & 4.8203 & \multirow{2}{*}{ Arch rib } & 1st order torsional \\
\hline 5.4851 & 5.5923 & -1.95 & 5.9617 & 5.8306 & & 2nd order torsional \\
\hline
\end{tabular}


TABLE 5: The MAC values of each model updating method.

\begin{tabular}{|c|c|c|c|}
\hline Mode shape & FE model & Kriging model & GA \\
\hline Arch rib 1st order symmetrical lateral bending & 0.9673 & 0.9678 & 0.9579 \\
\hline Girder 1st order antisymmetrical bending & 0.9931 & 0.9932 & 0.9932 \\
\hline Arch rib 1 st order antisymmetrical vertical bending & 0.8185 & 0.8173 & 0.8193 \\
\hline Girder 1st order antisymmetrical vertical bending & 0.9931 & 0.9932 & 0.9932 \\
\hline Arch rib 1 st order torsional & 0.9796 & 0.9791 & 0.9791 \\
\hline
\end{tabular}

TABle 6: Comparison of the error 2-norm.

\begin{tabular}{lcccccc}
\hline Initial model & Kriging model 1 & Kriging model 2 & Kriging model 3 & Kriging model 4 & Kriging model & GA \\
\hline 1.5215 & 1.6890 & 1.6019 & 1.3656 & 1.3468 & 1.0505 & 0.9852 \\
\hline
\end{tabular}

Note. Kriging model 1 takes the corresponding frequency of girder lateral mode shapes as the training samples; Kriging model 2 takes the corresponding frequency of arch rib torsional mode shapes as the training samples; Kriging model 3 takes the corresponding frequency of the girder arch rib vertical mode shapes as the training samples; Kriging model 4 takes the corresponding frequency of girder lateral mode shapes as the training samples; Secondary updated Kriging model is based on the calculation accuracy of the previous 4 Kriging models.

improves the calculation efficiency without sacrificing the accuracy.

The updated results by using GA show that only the error of first-order natural frequency is over $10 \%$, and the updated results have been improved a lot (its error 2-norm is smallest). Moreover, as enough population and genetic algebra are defined, GA can obtain higher accurate results, while the process takes a long time.

\section{Conclusions}

In this paper, a dynamic model updating procedure for bridges based on the Kriging model is presented. The Kriging model was established based on LHS, and the effectiveness of the Kriging model method was investigated in two typical bridges. Comparing the proposed method with $\mathrm{GA}$, the following conclusions can be drawn:

(1) After the FE model updating, the accuracy of modal frequencies and mode shapes is greatly improved. Therefore, the updated FE model can be used for a long-time structural health monitoring.

(2) The updated FE model with the Kriging model just needs a certain number of measured natural frequencies, and then a high accurate updated model can be obtained. In terms of computational cost reduction, compared to GA, the proposed method can reduce the computational cost without losing accuracy, which implies further potential application in the engineering field.

(3) The proposed method only applies to a truss bridge and a tied-arch bridge in the paper, and although the Kriging model has its advantages in reducing the computational expense compared to GA, the advantages of the proposed method should be further validated for the application in complex bridge structures, such as cable-stayed bridges, suspension bridges, and composite bridges.

(4) The proposed method is verified on a truss bridge and a tie-arch bridge; for the other types of bridges, such as simple supported beam bridge, suspension bridge, and cable-stayed bridge, the application can be done in the same manner.

\section{Data Availability}

The datasets generated and analyzed during the current study are available from the corresponding author upon reasonable request.

\section{Conflicts of Interest}

The authors declare that there are no conflicts of interest regarding the publication of this paper.

\section{Acknowledgments}

The first author wishes to thank the support from National Natural Science Foundation of China Grants nos. 11672108, 11202080 , and 51478193 and the innovation fund of South China University of Technology leading to an excellent Ph.D. dissertation. Shiping Huang is also supported by the Research Fund of The State Key Laboratory of Coal Resources and Safe Mining, CUMT, and by the Fundamental Research Funds for the Central Universities. The authors wish to thank all colleagues for helping in the survey and measurements. The support of China Railway Guangzhou Bureau Group Co., Ltd., Research Project is also gratefully appreciated.

\section{References}

[1] X. Kong and J. Li, "Vision-based fatigue crack detection of steel structures using video feature tracking," Computer-Aided Civil and Infrastructure Engineering, vol. 33, no. 9, pp. 783799, 2018.

[2] P. Asadollahi, Y. Huang, and J. Li, "Bayesian finite element model updating and assessment of cable-stayed bridges using wireless sensor data," Sensors, vol. 18, no. 9, pp. 1-22, 2018.

[3] S. G. Shahidi and S. N. Pakzad, "Generalized response surface model updating using time domain data," Journal of Structural Engineering, vol. 140, no. 8, article A4014001, 2014.

[4] Y. X. Zhang, C. X. Jia, J. Li, and B. F. Spencer, "Model updating based on an affine scaling interior optimization 
algorithm," Engineering Optimization, vol. 45, no. 11, pp. 1379-1395, 2012.

[5] A. M. Kabe, "Stiffness matrix adjustment using mode data," AIAA Journal, vol. 23, no. 9, pp. 1431-1436, 1985.

[6] P. D. Cha and W. Gu, "Model updating using an incomplete set of experimental modes," Journal of Sound and Vibration, vol. 233, no. 4, pp. 583-596, 2000.

[7] C. Minas and D. J. Inman, "Matching finite element models to modal data," Journal of Vibration and Acoustics, vol. 112, no. 1, pp. 84-92, 1990.

[8] D. C. Zimmerman and M. Widengren, "Correcting finite element models using a symmetric eigenstructure assignment technique," AIAA Journal, vol. 28, no. 9, pp. 1670-1676, 1990.

[9] S. Weng, Y. Xia, Y. L. Xu, X. Q. Zhou, and H. P. Zhu, "Improved substructuring method for eigensolutions of largescale structures," Journal of Sound and Vibration, vol. 323, no. 3-5, pp. 718-736, 2009.

[10] S. Q. Qin, Y. Z. Zhang, Y. L. Zhou, and J. Kang, "Dynamic model updating for bridge structures using the kriging model and PSO algorithm ensemble with higher vibration modes," Sensors, vol. 18, no. 6, p. 1879, 2018.

[11] T. Marwala, "Finite element model updating using response surface method," in Proceedings of Collection of Technical Papers-AIAA/ASME/ASCE/AHS/ASC Structures, Structural Dynamics and Materials Conference, Palm Springs, CA, USA, April 2004.

[12] W. X. Ren and H. B. Chen, "Finite element model updating in structural dynamics by using the Response surface method," Engineering Structures, vol. 32, no. 8, pp. 2455-2465, 2010.

[13] D. Shan, Q. Li, I. Khan, and X. Zhou, "A novel finite element model updating method based on substructure and response surface model," Engineering Structures, vol. 103, pp. 147-156, 2015.

[14] D. G. Krige, "A statistical approach to some mine valuation and allied problems on the Witwatersrand," M.S. thesis, University of the Witwatersrand, Johannesburg, South Africa, 1951.

[15] J. Sacks, S. B. Schiller, and W. J. Welch, "Designs for computer experiments," Technometrics, vol. 31, no. 1, pp. 41-47, 1989.

[16] V. J. Romero, L. P. Swiler, and A. A. Giunta, "Construction of response surfaces based on progressive-lattice-sampling experimental designs with application to uncertainty propagation," Structural Safety, vol. 26, no. 2, pp. 201-219, 2004.

[17] J. Zhang and F. T. K. Au, "Calibration of initial cable forces in cable-stayed bridge based on kriging approach," Finite Elements in Analysis and Design, vol. 92, pp. 80-92, 2014.

[18] L. Zhang, Z. Lu, and P. Wang, "Efficient structural reliability analysis method based on advanced kriging model," Applied Mathematical Modelling, vol. 39, no. 2, pp. 781-793, 2015.

[19] X. Huang, J. Chen, and H. Zhu, "Assessing small failure probabilities by AK-SS: an active learning method combining kriging and subset simulation," Structural Safety, vol. 59, pp. 86-95, 2016.

[20] J. T. Wang, C. J. Wang, and J. P. Zhao, "Frequency response function-based model updating using kriging model," $M e$ chanical Systems and Signal Processing, vol. 87, pp. 218-228, 2017.

[21] Y. C. Huang and K. Y. Chan, "A modified efficient global optimization algorithm for maximal reliability in a probabilistic constrained space," Journal of Mechanical Design, vol. 132, no. 6, article $061002,2010$.

[22] B. S. Kim, Y. B. Lee, and D. H. Choi, "Comparison study on the accuracy of metamodeling technique for non-convex functions," Journal of Mechanical Science and Technology, vol. 23, no. 4, pp. 1175-1181, 2009.

[23] V. Picheny, D. Ginsbourger, O. Roustant, R. T. Haftka, and N. H. Kim, "Adaptive designs of experiments for accurate approximation of a target region," Journal of Mechanical Design, vol. 132, no. 7, article 071008, 2010.

[24] B. Echard, N. Gayton, and M. Lemaire, "AK-MCS: AK-MCS: an active learning reliability method combining kriging and monte carlo simulation," Structural Safety, vol. 33, no. 2, pp. 145-154, 2011.

[25] B. J. Bichon, M. S. Eldred, L. P. Swiler, S. Mahadevan, and J. M. McFarland, "Efficient global reliability analysis for nonlinear implicit performance functions," AIAA Journal, vol. 46, no. 10, pp. 2459-2468, 2008.

[26] C. E. Rasmussen, "Gaussian processes in machine learning," Advanced Lectures on Machine Learning, vol. 3176, pp. 63-71, 2004.

[27] G. G. Wang and T. Simpson, "Fuzzy clustering based hierarchical metamodeling for design space reduction and optimization," Engineering Optimization, vol. 36, no. 3, pp. 313-335, 2011.

[28] C. Luo, K. Shimoyama, and S. Obayashi, "A study on manyobjective optimization using the kriging-surrogate-based evolutionary algorithm maximizing expected hypervolume improvement," Mathematical Problems in Engineering, vol. 2015, Article ID 162712, 15 pages, 2015.

[29] A. Wymyslowski, G. Q. Zhang, W. D. van Driel, and L. J. Ernst, "Virtual thermo-mechanical prototyping of microelectronics and microsystems," in Micro- and OptoElectronic Materials and Structures: Physics, Mechanics, Design, Reliability, Packaging, pp. A205-A266, Springer, Berlin, Germany, 2007.

[30] J. Wu, Q. S. Yan, J. Li, and M. Y. Hu, "Geometry control of long-span continuous girder concrete bridge during construction through finite element model updating," in Proceedings of Health Monitoring of Structural and Biological Systems, vol. 9805, Las Vegas, NV, USA, April 2016.

[31] A. Xu and R. L. Buchanan, "Evaluation of sampling methods for the detection of pathogenic bacteria on pre-harvest leafy greens," Food Microbiology, vol. 77, pp. 137-145, 2019.

[32] H. Samawi, A. Chatterjee, J. Yin, and H. Rochani, "On kernel density estimation based on different stratified sampling with optimal allocation," Communications in Statistics-Theory and Methods, vol. 46, no. 22, pp. 10973-10990, 2016.

[33] S. Makela, Y. Si, and A. Gelman, "Bayesian inference under cluster sampling with probability proportional to size," Statistics in Medicine, vol. 37, no. 26, pp. 3849-3868, 2018.

[34] F. F. Kao, C. H. Leu, and C. H. Ko, "Remainder markov systematic sampling," Journal of Statistical Planning and Inference, vol. 141, no. 11, pp. 3595-3604, 2011.

[35] M. D. McKay, R. J. Beckman, and W. J. Conover, "Comparison of three methods for selecting values of input variables in the analysis of output from a computer code," Technometrics, vol. 21, no. 2, pp. 239-245, 1979.

[36] R. L. Iman, J. C. Helton, and J. E. Campbell, “An approach to sensitivity analysis of computer models: Part I-introduction, input variable selection and preliminary variable assessment," Journal of Quality Technology, vol. 13, no. 3, pp. 174-183, 1981.

[37] M. Petelet, B. Iooss, O. Asserin, and A. Loredo, "Latin hypercube sampling with inequality constraints," AStA Advances in Statistical Analysis, vol. 94, no. 4, pp. 325-339, 2011. 
[38] S. N. Lophaven, H. B. Nielsen, and J. Søndergaard, Dace: A Matlab Kriging Toolbox, Technical University of Denmark, Lyngby, Denmark, 2002.

[39] G. R. Yan, Z. D. Duan, and J. P. Ou, "Application of genetic algorithm on structural finite element model updating," Journal of Harbin Institute of Technology, vol. 39, no. 2, pp. 181-186, 2007.

[40] https://www.ansys.com/products. 


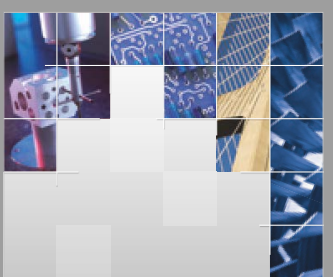

\section{Enfincering}
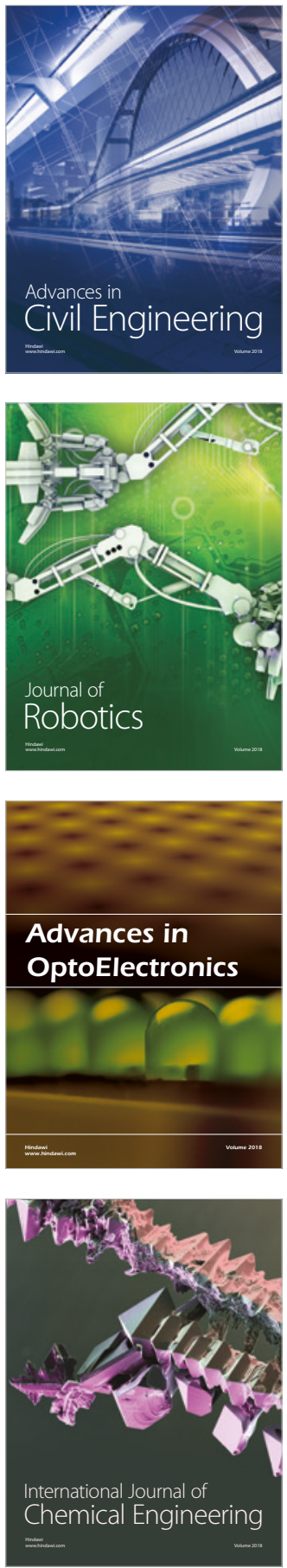

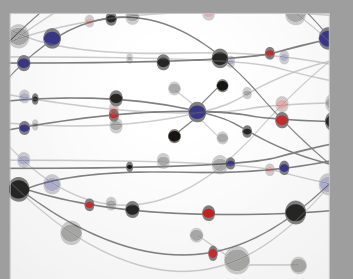

\section{Rotating \\ Machinery}

The Scientific World Journal

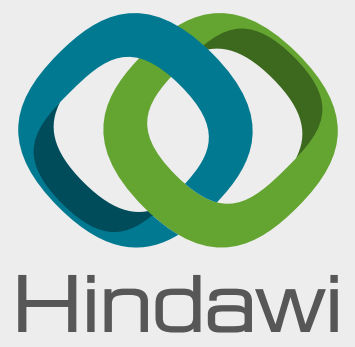

Submit your manuscripts at

www.hindawi.com
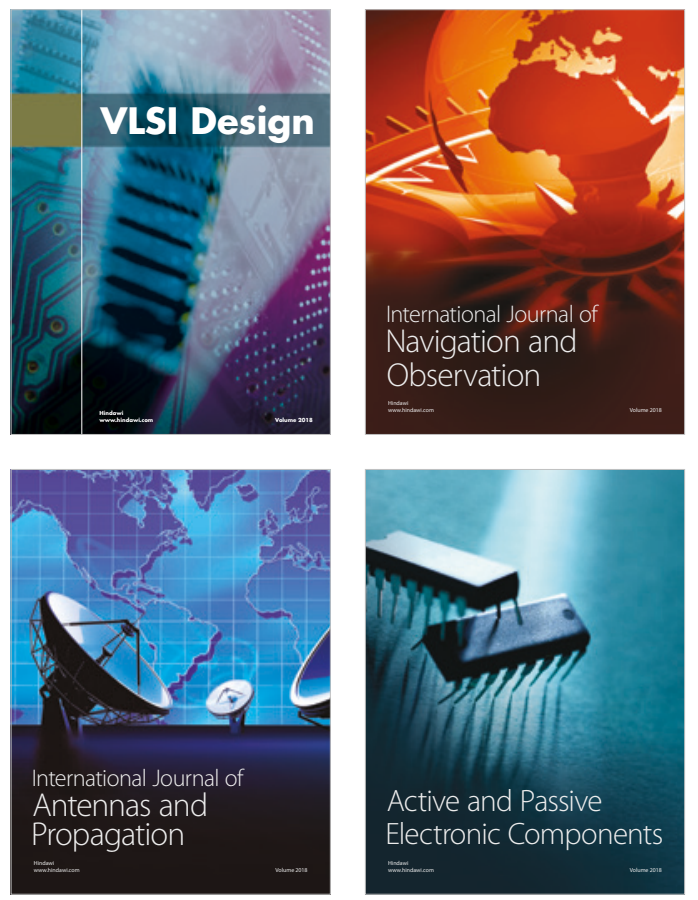
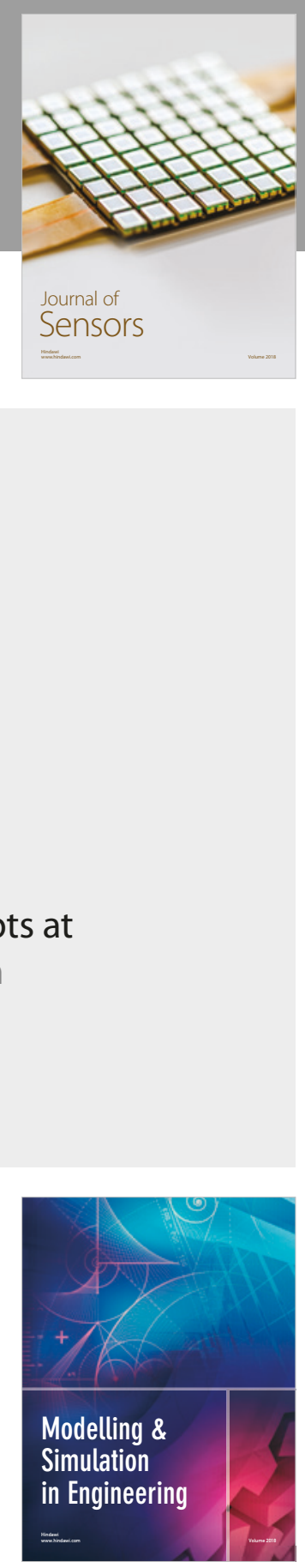

\section{Advances \\ Multimedia}
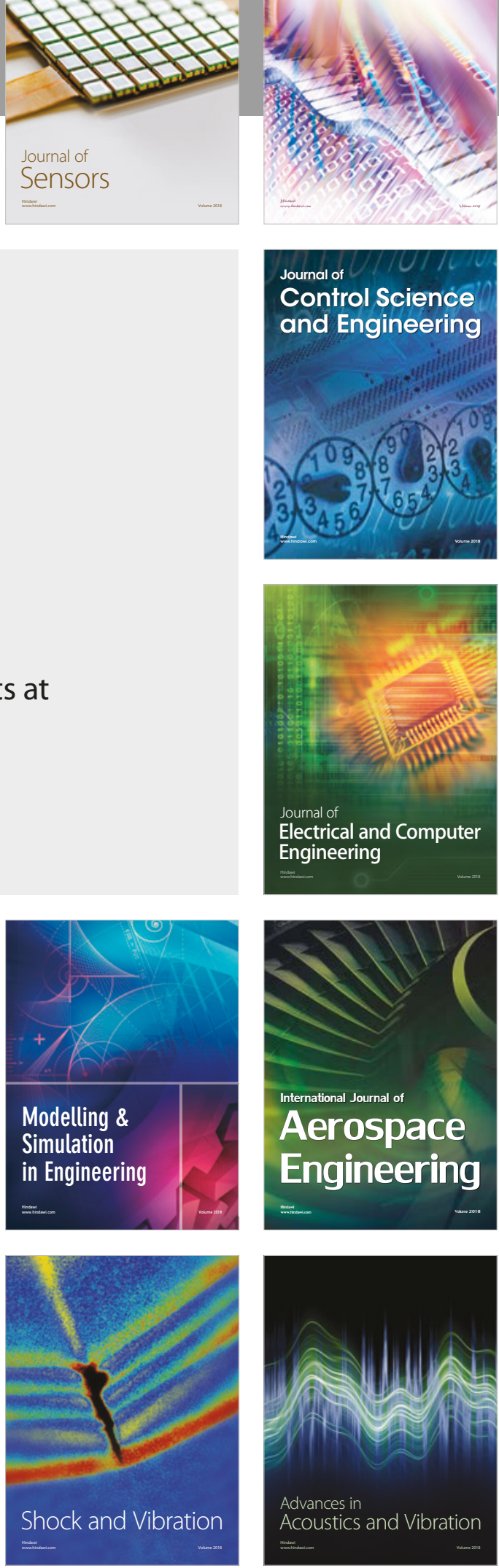